\title{
DIRECT PRODUCT DECOMPOSITION OF COMMUTATIVE SEMISIMPLE RINGS
}

\section{ALEXANDER ABIAN}

Abstract. In this paper it is shown that a commutative semisimple ring is isomorphic to a direct product of fields if and only if it is hyperatomic and orthogonally complete.

In this paper we give a necessary and sufficient condition for a commutative semisimple ring $R$ (i.e., $R$ has no nonzero nilpotent element) to be isomorphic to a direct product of fields. In particular, we show that hyperatomicity and orthogonal completeness is such a necessary and sufficient condition. It is well known that without these conditions $R$ is isomorphic to a subring of a direct product of fields $[1$, p. 16].

We would like to emphasize that, in what follows, $R$ stands for a commutative ring with no nonzero nilpotent element. Thus, in particular, for every element $x$ of $R$,

$$
x^{2}=0 \text { if and only if } x=0 .
$$

We first prove several lemmas. Lemma 1 below, generalizes the corresponding result for Boolean Rings [2, p. 154].

Lemma 1. The ring $R$ is partially ordered by $\leqq$ where for every element $x$ and $y$ of $R$,

$$
x \leqq y \text { if and only if } x y=x^{2} .
$$

Proof. Since $x x=x^{2}$ it follows from (2) that $x \leqq x$. Thus, $\leqq$ is reflexive.

Moreover, if $x \leqq y$ and $y \leqq x$, then $x y=x^{2}$ and $y x=y^{2}$ so that $x^{2}-x y$ $-y x+y^{2}=(x-y)^{2}=0$. But then $x-y=0$ by (1). Thus, $x=y$ and therefore $\leqq$ is antisymmetric.

Furthermore, if $x \leqq y$ and $y \leqq z$, then $x y=x^{2}$ and $y z=y^{2}$ so that $x^{2} z=x y z=x y^{2}=x^{2} y=x^{3}$. Thus, $x^{2} z^{2}=x^{3} z$ and $x^{3} z=x^{4}$ so that $x^{2} z^{2}-x^{3} z$ $-x^{3} z+x^{4}=\left(x z-x^{2}\right)^{2}=0$. But then from (1) it follows that $x z-x^{2}=0$ or $x z=x^{2}$. Hence, $x \leqq z$ by (2), and therefore, $\leqq$ is transitive.

In the following the symbol $\leqq$ is used only to represent the partial order defined by (2).

Received by the editors June 30, 1969.

A MS Subject Classifications. Primary 1350, 1644, 1650.

Key Words and Phrases. Decomposition as direct product, commutative semisimple ring, decomposition as complete direct sum, commutative ring, nonzero nilpotent element. 
Let us observe that from (2) it follows immediately that for every element $x, y$ and $z$ of $R$

$$
x \leqq y \text { implies } x z \leqq y z
$$

and

$$
x^{2}=x \text { implies } x y \leqq y .
$$

Motivated by $[2$, p. 7], we introduce the following definition.

Definition 1. A nonzero element a of $R$ is called a hyperatom of $R$ if and only if for every element $x$ of $R$

$$
x \leqq a \text { implies } x=0 \text { or } x=a
$$

and

$$
a x \neq 0 \text { implies axs }=a
$$

for some element $s$ of $R$.

Next, we prove

Lemma 2. Let a be a hyperatom of $R$. For every element $r$ of $R$ if ar $\neq 0$ then ar is a hyperatom of $R$.

Proof. Let $a r \neq 0$. We show that $a r$ is a hyperatom according to Definition 1.

Since $a r \neq 0$, by (6) we have $a r s=a$ for some element $s$ of $R$. Now, let $x \leqq a r$ then from (3) it follows that $x s \leqq a r s$. Hence $x s \leqq a$, and in view of (5) we have

$$
x s=0 \text { or } x s=a .
$$

However, $x \leqq a r$ so that $a r x=x^{2}$ and therefore $r s x^{2}=r s(a r x)$ $=(r s a) r x=a r x=x^{2}$. Consequently, $(r s x-x)^{2}=(r s)^{2} x^{2}-2 r s x^{2}+x^{2}=0$. Thus, by (1) we have $r s x=x$ which in view of the above implies that $x=0$ or $x=a r$. Hence $a r$ satisfies (5).

On the other hand, if $a r x \neq 0$ then there exists an element $t$ of $R$ such that $a r x t=a$. Thus, $(a r x) t r=a r$, so that $a r$ satisfies (6).

In view of the above two cases, we see that $a r$ is a hyperatom of $R$, as desired.

Lemma 3. Let a be a hyperatom of $R$. Then there exists an element $s$ of $R$ such that as is an idempotent hyperatom of $R$.

Proof. Since $a \neq 0$ it follows from (1) that $a^{2} \neq 0$. Thus, by (6), there exists an element $s$ of $R$ such that $a^{2} s=a$. Clearly, as $\neq 0$ and therefore $a s$ is a hyperatom by Lemma 2 . But also, $(a s)^{2}=\left(a^{2} s\right) s=a s$. Thus, $a s$ is an idempotent hyperatom of $R$. 
Definition 2. A subset $S$ of $R$ is called orthogonal if and only if $x y=0$ for every two distinct elements $x$ and $y$ of $S$.

Lemma 4. The set $\left(e_{i}\right)_{i \in I}$ of all idempotent hyperatoms of $R$ is an orthogonal set.

Proof. Let $e_{i}$ and $e_{j}$ be idempotent hyperatoms of $R$. From (4) it follows that $e_{i} e_{j} \leqq e_{i}$ and $e_{i} e_{j} \leqq e_{j}$ so that $e_{i} e_{j}=e_{i}=e_{j}$ or $e_{i} e_{j}=0$.

Lemma 5. Let $\left(e_{i}\right)_{i \in I}$ be the set of all idempotent hyperatoms of $R$. Then for every element $i$ of $I$ the ideal

$$
F_{i}=\left\{r e_{i} \mid r \in R\right\}
$$

is a subfield of $R$ and

$$
F_{i} \cap F_{j}=\{0\} \quad \text { if } i \neq j .
$$

Proof. Since $e_{i}^{2}=e_{i}$ it follows that $e_{i}$ is an element of $F_{i}$ and for every element $r$ of $R$ we have $\left(r e_{i}\right) e_{i}=r e_{i}$ so that $e_{i}$ is the unit of $F_{i}$. Further, if $r e_{i} \neq 0$ then since $e_{i}$ is a hyperatom there exists an element $s$ of $R$ such that $\left(s e_{i}\right)\left(r e_{i}\right)=s r e_{i}=e_{i}$. Thus, each nonzero element of $F_{i}$ has an inverse in $F_{i}$ so that $F_{i}$ is a field.

Finally, if $i \neq j$ and $r e_{i}=s e_{j}$ then $r e_{i} e_{j}=s e_{j} e_{j}=s e_{j}=r e_{i}=0$ since $e_{i} e_{j}=0$. Thus, $F_{i} \cap F_{j}=\{0\}$.

Definition 3. The ring $R$ is called hyperatomic if and only if for every nonzero element $r$ of $R$ there exists a hyperatom a of $R$ such that $a \leqq r$.

In the following sup $S$, for a subset $S$ of $R$, is used in reference to the order given by (2).

Lemma 6. Let $R$ be hyperatomic and let $\left(e_{i}\right)_{i \in I}$ be the set of all idempotent hyperatoms of $R$. Then for every nonzero element $q$ of $R$ there exists an idempotent hyperatom $e_{k}$ such that $q e_{k} \neq 0$. Moreover, for every element $r$ of $R$ the sup $_{i} r_{i}$ exists and

$$
r=\sup _{t} r e_{i}
$$

Proof. By Definition 3 if $q \neq 0$ there is a hyperatom $a$ of $R$ such that $a \leqq q$ or $q a=a^{2} \neq 0$. But then by Lemma 3 there exists an element $s$ of $R$ such that as is an idempotent hyperatom. Clearly, $q(a s) \neq 0$. Hence $q e_{k} \neq 0$ for some idempotent hyperatom $e_{k}=a s$ of $R$.

To prove the second assertion we note that $r\left(r e_{i}\right)=\left(r e_{i}\right)^{2}$ for every element $i$ of $I$ so that $r$ is an upper bound of the set $\left(r e_{i}\right)_{i \in I}$. Let $u$ be any upper bound of $\left(r e_{i}\right)_{i \in I}$. Then $u r e_{i}=\left(r e_{i}\right)^{2}=r r e_{i}$ for every element $i$ of $I$. We claim that $r \leqq u$. Assume on the contrary, that $u r-r^{2}$ $=q \neq 0$. Thus, in view of the above, for some element $k$ of $I$ it is the 
case that $u r e_{k}-r^{2} e_{k}=q e_{k} \neq 0$. But this contradicts that $u r e_{i}=r r e_{i}$ for every element $i$ of $I$. Thus, indeed $r=\sup _{i} r e_{i}$. Hence, the lemma is proved.

If $\left(e_{i}\right)_{i \in I}$ is the set of all idempotent hyperatoms of $R$, then by (8) we may consider the direct product $\prod_{i} F_{i}$ of the fields $F_{i}$ given by (7). We then have the following

Lemma 7. Let $R$ be hyperatomic and let $\left(e_{i}\right)_{i \in I}$ be the set of all idempotent hyperatoms of $R$. Then

$$
\alpha(r)=\left(r e_{i}\right)_{i \in I}
$$

is an isomorphism from $R$ onto a subring of the direct product $\Pi_{i} F_{i}$ of fields $F_{i}$.

Proof. Clearly, $\alpha$ is a homomorphism so it suffices to show that $\alpha$ is one-to-one. But if $r \neq q$ it follows from (9) that $\sup _{\boldsymbol{i}} r e_{\boldsymbol{i}} \neq \sup _{\boldsymbol{i}} q e_{\boldsymbol{i}}$ so that $\left(r e_{i}\right)_{i \in I} \neq\left(q e_{i}\right)_{i \in I}$ or $\alpha(r) \neq \alpha(q)$. Hence, $\alpha$ is an isomorphism.

As mentioned earlier the existence of an isomorphism from $R$ to a subring of a direct product of fields is well known and requires no additional conditions on $R$. However, the special isomorphism $\alpha$ given in Lemma 7 is essential for the proof of the theorem below which states that hyperatomicity and orthogonal completeness of $R$ is a necessary and sufficient condition for $R$ to be isomorphic to a direct product of fields.

One additional lemma is also needed, but first we observe that if $\sup _{i} r_{i}$ exists for a subset $\left(r_{i}\right)_{i \in I}$ of $R$ then $r_{i} \leqq \sup _{i} r_{i}$, so that by (2) we have

$$
r_{i} \sup _{i} r_{i}=r_{i}^{2}
$$

Lemma 8. Let $\left(r_{i}\right)_{i \in I}$ be a subset of $R$ such that $\sup _{i} r_{i}$ exists. Then for every element $b$ of $R$ the sup $_{i} b r_{i}$ exists and

$$
b \sup _{i} r_{i}=\sup _{i} b r_{i} \text {. }
$$

Proof. From (11) it follows that $\left(b r_{i}\right)\left(b \sup _{i} r_{i}\right)=\left(b r_{i}\right)^{2}$ so that $b r_{i} \leqq b \sup _{i} r_{i}$ for every element $i$ of $I$. Thus, $b$ sup $_{i} r_{i}$ is an upper bound of the set $\left(b r_{i}\right)_{i \in I}$.

Let $u$ be any upper bound of $\left(b r_{i}\right)_{i \in I}$. Then $u b r_{i}=\left(b r_{i}\right)^{2}$ for every element $i$ of $I$. Thus, $u b r_{i}-b^{2} r_{i}^{2}+r_{i}^{2}=r_{i}^{2}$. But then from (11) we see that for every element $i$ of $I$

$$
r_{i}\left(u b-b^{2} \sup _{i} r_{i}+\sup _{i} r_{i}\right)=r^{2}
$$


so that

$$
r_{i} \leqq u b-b^{2} \sup _{i} r_{i}+\sup _{i} r_{i}
$$

and therefore

$$
\sup _{i} r_{i} \leqq u b-b^{2} \sup _{i} r_{i}+\sup _{i} r_{i} .
$$

Consequently, it follows from (2) that

$$
\left(\sup _{i} r_{i}\right)\left(u b-b^{2} \sup _{i} r_{i}+\sup _{i} r_{i}\right)=\left(\sup _{i} r_{i}\right)^{2}
$$

which implies that

$$
u b \sup _{i} r_{i}=\left(b \sup _{i} r_{i}\right)^{2}
$$

and therefore,

$$
b \sup _{i} r_{i} \leqq u .
$$

Hence, $\sup _{i} b r_{i}$ exists and is equal to $b \sup _{i} r_{i}$.

DEFINITION 4. The ring $R$ is called orthogonally complete if and only if $\sup S$ of every orthogonal subset $S$ of $R$ exists.

Finally, we have

ThEOREM. The ring $R$ is isomorphic to a direct product of fields if and only if $R$ is hyperatomic and orthogonally complete.

Proof. Let $\beta$ be an isomorphism from $R$ onto a direct product $\prod_{i \in I} K_{i}$ of fields $K_{i}$. Let $r$ be a nonzero element and let $\beta(r)=\left(r_{i}\right)_{i \in I}$. There exists an element $j$ of $I$ such that $r_{j} \neq 0$. Let $u_{j}$ be the unit of $K_{j}$. The element $a$ of $R$ given by $a=r \beta^{-1}\left(\left(k_{i}\right)_{i \in I}\right)$ with $k_{j}=u_{j}$ and $k_{i}=0$ for $i \neq j$ is obviously a hyperatom of $R$ with $a \leqq r$. Hence, $R$ is hyperatomic.

Next, let $S$ be an orthogonal subset of $R$ and let $\beta[S]$ $=\left(\left(k_{i}(s)\right)_{i \in I}\right)_{s \in S}$. Since $S$ is orthogonal it follows that $\beta^{-1}\left(\left(k_{i}\right)_{i \in I}\right)$ $=\sup S$ where $k_{i}=k_{i}(s)$ if $k_{i}(s) \neq 0$ for some element $s$ of $S$ and $k_{i}=0$ otherwise. Thus, $R$ is orthogonally complete.

Conversely, if $R$ is hyperatomic and orthogonally complete we show that $R$ is isomorphic to the direct product $\prod_{i \in I} F_{i}$ of fields $F_{i}$ given by (7). In view of Lemma 7 it will suffice to show that the mapping $\alpha$ of (10) is an onto mapping. Let $\left(r_{i} e_{i}\right)_{i \in I}$ be any element of $\prod_{i} F_{i}$. By Lemma 4 the set $\left(r_{i} e_{i}\right)_{i \in I}$ is an orthogonal subset of $R$. Let $h=\sup _{i} r_{i} e_{i}$. But then from (12) and Lemma 4 it follows that for every element $j$ of $I$ 


$$
h e_{j}=e_{j} \sup _{i} r_{i} e_{i}=\sup _{i} r_{i} e_{i} e_{j}=r_{j} e_{j} .
$$

Hence, by (10) we have $\alpha(h)=\left(h e_{i}\right)_{i \in I}=\left(r_{i} e_{i}\right)_{i \in I}$ so that $\alpha$ is an onto mapping. Thus, the theorem is proved.

\section{REFERENCES}

1. N. Jacobson, Structure of rings, Amer. Math. Soc. Colloq. Publ., vol. 37, Amer. Math. Soc., Providence, R. I., 1956. MR 18, 373.

2. Garrett Birkhoff, Lattice theory, Amer. Math. Soc. Colloq. Publ., vol. 25, Amer. Math. Soc., Providence, R. I., 1934; 2nd rev. ed., 1967. MR 37 \#2638.

Iowa State University 